\title{
GENERACIÓN AUTOMÁTICA DE PLANOS ESTRUCTURALES A TRAVÉS DE PCS
}

\section{(AUTOMATIC GENERATION OF STRUCTURAL DRAWINGS USING PCS)}

\author{
Jesús Chomón Díaz, Dr. Ingeniero Industrial \\ Eduardo Gómez López, Dr. Ingeniero de Caminos. \\ Profs. de la E.T.S. de Arquitectura.
}

UNIVERSIDAD POLITÉCNICA DE MADRID, ESPAÑA

\section{RESUMEN}

Se realiza una breve descripción de la evolución, tanto en el aspecto del software como del hardware, en el mundo del ordenador personal, el PC. Se define como perfectamente realizable la generación automática de planos utilizando estos equipos, haciendo hincapié en el aspecto del software, módulos de entrada de datos, cálculo, armado y dibujo. Sobre este último se profundiza, para el caso especifico de Autocad, y se explica la generación de ficheros DXF.

Se acompañan dos anexos, con la programación realizada en QuickBasic, para la generación de una librería de dibujo y de un programa de volcado automático de una serie de planos a plotter.

Igualmente se presentan ejemplos de salida impresa de ordenador y plotter.

\section{SUMMARY}

This is a brief description of the evolution, of sofware as well as hardware, in the world of personal computer, PC. It has thoroughly put into practice the automatic generation of drawings using this equipment, emphasizing in the software, data input moduli, calculations, reinforcement and drawing. In the last item it's deepened for Autocad specifically and it has explained the DXF files generation.

Attached are two addenda, with the programs made in QuickBasic, for the generation of a drawing library, and another program of automatic output of a group of drawings to a plotter.

In the same way are presented some examples of printer and plotter out-puts.

\section{INTRODUCCIÓN}

Hace años, era un logro notable, sobre todo desde un modesto ordenador personal, disponer de un programa de cálculo de estructuras capaz de obtener esfuerzos en barras y, como mucho, el área de armadura de refuerzo necesaria para estructuras de hormigón armado.

Pero en pocos años, la evolución en el campo informático ha sido enorme, tanto en el aspecto del software como del hardware, para equipos multiusuarios de gran potencia y para equipos asequibles tanto por precio como por facilidad de uso, como es el caso de los PC's.

Por ello, es perfectamente realizable hoy día obtener, a través de las envolventes de esfuerzos, el armado de elementos estructurales o de la estructura completa, generando los planos de un modo automático, de for- ma que se pueda, si es necesario, modificar los planos inicialmente generados por el ordenador mediante un programa o sistema de CAD. Al mismo tiempo se puede obtener una medición de la estructura, al estar completamente definida en el ordenador.

Sobre estos equipos, genéricamente denominados PC's como si de un modelo único se tratara vamos a centrarnos en lo sucesivo, observando que suponen una plataforma de hardware muy adecuada, sobre la que existe una gran cantidad de software desarrollado, en ocasiones de muy alta calidad, sobre el que si se considera necesario se puede implementar el software propio.

Para la generación automática de planos lo normal es apoyarse en un sistema de CAD de los existentes en el mercado. Nuestros desarrollos han sido realizados sobre Autocad, uno de los más difundidos. 
El equipo necesario para realizar la tarea propuesta se compone de uno o varios PC's, con pantalla gráfica en color para tareas de cálculo y delineación y un plotter.

\section{SOFTWARE ESPECÍFICO}

El software, o programas que han de realizar la tarea de cálculo y preparación de planos, puede consistir en un desarrollo propio adecuado a las necesidades específicas del usuario, o bien puede ser adquirido si se encuentra un programa que cubra esas necesidades.

En cualquier caso un programa de cálculo de estructuras que tenga como objetivo último la generación automática de planos, y casi siempre la obtención de la medición de acero y hormigón, se compondrá de una serie de módulos básicos:

a) Un módulo inicial que tenga como objetivo la entrada de los datos que definan la estructura, así como su modificación tan frecuente en este tipo de cálculos, bien por defectos iniciales, o bien por reconsideración a la vista de los resultados obtenidos en un cálculo previo.

b) Un módulo de cálculo propiamente dicho que, basado en los datos de la estructura, realice, normalmente por métodos matriciales, la obtención de los desplazamientos de los nudos de la estructura. Partiendo de esos desplazamientos, se obtienen los esfuerzos en las secciones que se considere necesario, en vigas al menos en diez secciones por vano, en pilares normalmente en cabeza y pie de pilares.

c) Un tercer módulo de armado de cada uno de los elementos estructurales, normalmente vigas y pilares, teniendo en cuenta la envolvente de esfuerzos, momentos flectores y esfuerzos cortantes. Este módulo suele ser el encargado de la medición al mismo tiempo que se va obteniendo cada una de las armaduras de refuerzo necesarias.

d) Un cuarto y último módulo que se encarga de la elaboración del fichero de dibujo propiamente dicho. En el caso de utilizar posteriormente Autocad, este fichero es un ASCII, generado según los criterios de ficheros DXF, suficientemente explicado en los manuales de Autocad.

Los dos primeros módulos existen normalmente en cualquier programa de cálculo de estructuras, si bien el segundo ha de obtener los esfuerzos en número de secciones superior al normal, a fin de disponer de datos suficientes para poder decidir el punto de corte de las barras en el tercer módulo.

El tercer módulo, aunque no es todavía el módulo de dibujo, es el que debe contener los criterios de arma- do y, al obtener los refuerzos de armaduras necesarias, obtiene todos los datos necesarios para el módulo siguiente. Se trata de un módulo importantísimo, pues de la bondad de los algoritmos utilizados depende el que las armaduras obtenidas cumplan con los requisitos fundamentales de seguridad.

Este aspecto siempre fundamental en el cálculo de estructuras, lo es doblemente cuando el cálculo se realiza a través de ordenador, pues existe una tendencia a la aceptación de esos resultados que está claro que sólo serán tan buenos o tan malos como lo sea el programa que los genera. Pero con la utilización del ordenador y su uso en el cálculo de un gran número de estructuras, siempre con los mismos criterios, los del programa base, si éste está suficientemente depurado es una garantía de calidad, pero si contiene algún error importante, este error aparecerá sistemáticamente en todos los cálculos realizados, con la responsabilidad que ello supone. Por ello consideramos que estos programas deben ser siempre utilizados POR PROFESIONALES EXPERTOS, que sean capaces de detectar algún problema caso de que se presentara.

Este tercer módulo es, por tanto, muy importante. En él se deben decidir qué tipos de armaduras longitudinales se van a utilizar para resistir los momentos flectores más desfavorables que se produzcan en cualquiera de las combinaciones de hipótesis, así como los cercos que deben resistir los esfuerzos cortantes, pero siempre cumpliendo los dos requisitos anteriores, es decir, disponer las armaduras necesarias, las estrictamente necesarias, en el lugar donde haga falta.

\section{EL MÓDULO DE DIBUJO}

El cuarto módulo, el de dibujo, es el que se encarga de generar un fichero ASCII, en formato DXF para el caso de Autocad, para cada uno de los planos que se produzcan como consecuencia del cálculo realizado. Inicialmente se generará un fichero para el primer plano a dibujar. Sobre este fichero se irá grabando la información necesaria correspondiente a líneas, textos y todos los elementos gráficos en la posición adecuada.

Es muy conveniente preparar un módulo que permita, de un modo cómodo, acceder a la grabación del fichero DXF, que consta en primer lugar de una cabecera, pudiendo generar al mismo tiempo el recuadro o formato del plano sobre el que se va a dibujar. Este formato puede ser fijo, normalmente de un tamaño DIN A1 ó A0, o variable para cada proyecto, si éste tiene unos requerimientos especiales. Este módulo debe contener las subrutinas necesarias que se encarguen de la grabación en el fichero que contiene los datos del plano de aquellos comandos del paquete gráfico, Autocad en nuestro caso, necesarios para la representación del dibujo. Estas subrutinas básicamente son 
LÍNEA, POLILÍNEA, CÍRCULO, TEXTO, cada una de ellas con la posibilidad de definir parámetros como color, altura e inclinación para textos, etc.

A partir del módulo descrito en el párrafo anterior, el programa de dibujo, cada vez que se genere un nuevo plano, hará una llamada a la subrutina de cabecera, con lo que se abrirá el fichero que contiene la descripción del plano comenzando a grabar en él los datos generales, los de descripción del formato del plano, estando en condiciones de recibir la información de líneas y textos que formarán el plano en sí mismo. Es muy útil disponer de un sistema de coordenadas globales, cuyo origen puede estar situado en el ángulo inferior izquierdo del plano, y de un sistema de coordenadas específico de cada uno de los elementos a dibujar, relacionado con el sistema de coordenadas globales por un desplazamiento XDI, YDI. De este modo, aquellos elementos repetitivos a dibujar en el plano quedan situados en otro lugar diferente con sólo variar los valores adjudicados a las variables de desplazamiento XDI, YDI. Cada vez que se dibujan o, más exactamente, se graban en el fichero la información de dibujo mediante las Ilamadas a las subrutinas LÍNEA, POLILÍNEA, TEXTO, CÍRCULO, etc. es preciso contabilizar de algún modo que este espacio del plano ha sido ya utilizado, a fin de no dibujar sobre él otro elemento gráfico que quedaria montado sobre el anterior. De este modo el plano se irá llenando, con lo que en un momento determinado será preciso cerrar el fichero, dando por terminada la grabación de elementos de dibujo sobre el mismo. A continuación será necesario abrir un nuevo fichero de dibujo para continuar el proceso de cálculo, siendo preciso cerrarlo cuando éste finalice, independientemente de que el último plano esté o no completo.

Los ficheros de dibujo, generados con el nombre XXXX.DXF, pueden ser visualizados por Autocad mediante la orden DXFIN, con lo que se consigue transformar los ficheros ASCII generados por él o los programas de cálculo a ficheros binarios tipo XXXX.DWG, propios de Autocad, pudiendo desde este momento modificar los dibujos o volcarlos a plotter cuando se considere necesario.

En el apartado VI, ANEXO 1, se facilita un listado de una librería de dibujo, en QuickBasic, que contiene las siguientes subrutinas:

1) ENCAB.- Graba en el fichero de dibujo las cabeceras necesarias para Autocad. Tiene como parámetros de entrada DXP, DYP, las dimensiones en $\mathrm{cm}$ de los planos a generar, dibujando el recuadro.

2) LÍNEA.- Graba en el fichero de dibujo los datos correspondientes a la entidad línea. Tiene como parámetros de entrada:
IC.- Indica el color de la línea.

XDI. - Indica el desplazamiento en $X$, respecto a las coordenadas globales.

YDI. - Indica el desplazamiento en Y, respecto a las coordenadas globales.

ITS.- Es una variable tipo carácter que especifica el tipo de línea, continua, rayitas, etc.

$\mathrm{X} 1, \mathrm{Y} 1$. - Son las coordenadas locales del comienzo de la línea.

$\mathrm{X} 2, \mathrm{Y} 2$.- Son las coordenadas locales del final de la línea.

3) POLI.- Graba en el fichero de dibujo los datos correspondientes a la entidad Polilínea. Tiene como parámetros específicos:

NLI._ Indica el número de líneas que componen la polilínea.

KCIE.- Indica si la polilínea es cerrada (1) o no (0).

XP,YP. - Son las coordenadas locales de los vértices de la polilínea.

4) TEX.- Graba en el fichero de dibujo los datos correspondientes a textos horizontales, los más usuales. Sus parámetros particulares son:

HT.- Indica la altura del texto en $\mathrm{mm}$.

TS. - Es una variable tipo carácter, que contiene el texto a dibujar.

5) TEXIN.-Graba en el fichero de dibujo los datos correspondientes a textos inclinados. Sus parámetros son los de la orden TEX, más ANG, que indica el ángulo con que se desea que aparezca el texto.

6) CIRCUL.- Graba en el fichero de dibujo los datos correspondientes a círculos. Sus parámetros específicos son:

$\mathrm{X} 1, \mathrm{Y} 1 .-$ Coordenadas locales del centro del círculo. R.- Radio del círculo.

Puede ser interesante dar otro paso más en el proceso de automatización preparando un programa general, que permita la conversión de los ficheros DXF generados por el programa de cálculo, en los ficheros DWG tratables por Autocad, sin tener que entrar en este programa para convertir uno a uno los ficheros, con lo que este proceso se realiza de un modo automático y por lo tanto desatendido. Un ejemplo de ello se facilita en el apartado VII, ANEXO II, en el que se facilita un listado, también en el QuickBasic de un programa que permite la conversión de hasta 50 planos.

Este módulo parte de la base de que se van a automatizar la obtención de planos de vigas y de nervios, los primeros se denominan, y asi han sido generados por el programa de cálculo PVxxx, situados en el directorio VIGAS. Los ficheros de nervios han sido llamados PLXXX, situados en el directorio NERVIOS. 


\section{CONCLUSIÓN}

Se ha intentado describir, sin poder entrar en todos los detalles pertinentes, un proceso de un gran interés, como es el de la obtención de planos estructurales, directamente del cálculo realizado mediante ordenadores muy extendidos en la actualidad y de bajo coste, como son los PC's. Este proceso es muy interesante, en primer lugar por la alta rentabilidad que reporta, ya que evita el dibujo manual de estos planos que es una tarea larga y tediosa y, por lo tanto, cara. Pero, además, si el programa está suficientemente probado, es claro que proporciona una mayor seguridad, pues evita cualquier problema de transcripción de los valores de cálculo.

También es claro que el éxito de la implantación de un paquete de este tipo, se basa en que cubra, de un mo- do eficiente, las necesidades del grupo de trabajo que to ha de utilizar, debiendo participar si considera necesario el desarrollo de un paquete especifico, en su gestación y puesta a punto.

\section{BIBLIOGRAFÍA}

Autocad User Reference.- Autodesk AG. Autocad Avanzado V.10. - J. López Fernández y J. C. Bartomé Larrinaga.

Customizing Autocad.- J. Smith y R. Gesner.

AutoLISP 90:- Autodesk AG.

Microsoft QuickBASIC 4.0.- Microsoft Corporation: BASIC Language Reference.

Programming in BASIC: Selected Topics. Learning and Using Microsoft QuickBASIC.

\section{PROGRAMA}

VI) ANEXO I.

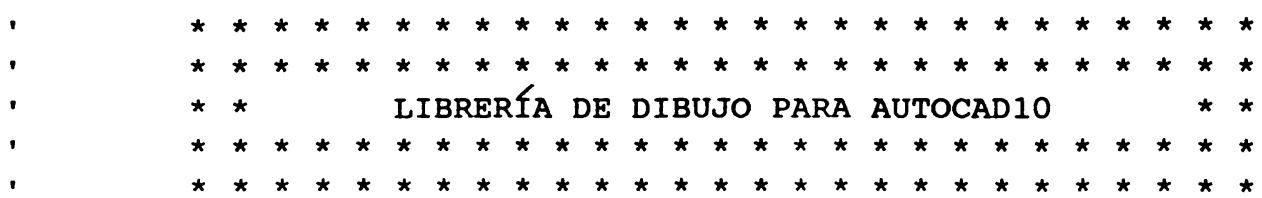

SUB ENCAB (DXP, DYP)

- ENCABEZAMIENTO DEL FIDXF.

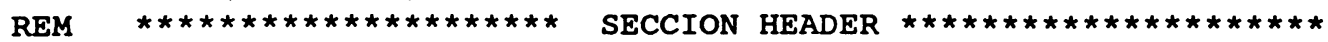

PRINT \#1, 0: PRINT \#1, "SECTION": PRINT \#1, 2: PRINT \#1, "HEADER"

PRINT \#1, 9: PRINT \#1, "\$ACADVER": PRINT \#1, 1: PRINT \#1, "AC1002"

PRINT \#1, 9: PRINT \#1, "\$INSBASE": PRINT \#1, 10: PRINT \#1, 0!: PRINT \#1, 20

PRINT \#1, 0 !

PRINT \#1, 9: PRINT \#1, "\$COORDS": PRINT \#1, 70: PRINT \#1, 1

PRINT \#1, 9: PRINT \#1, "\$EXTMIN": PRINT \#1, 10!: PRINT \#1, 0: PRINT \#1, 20 


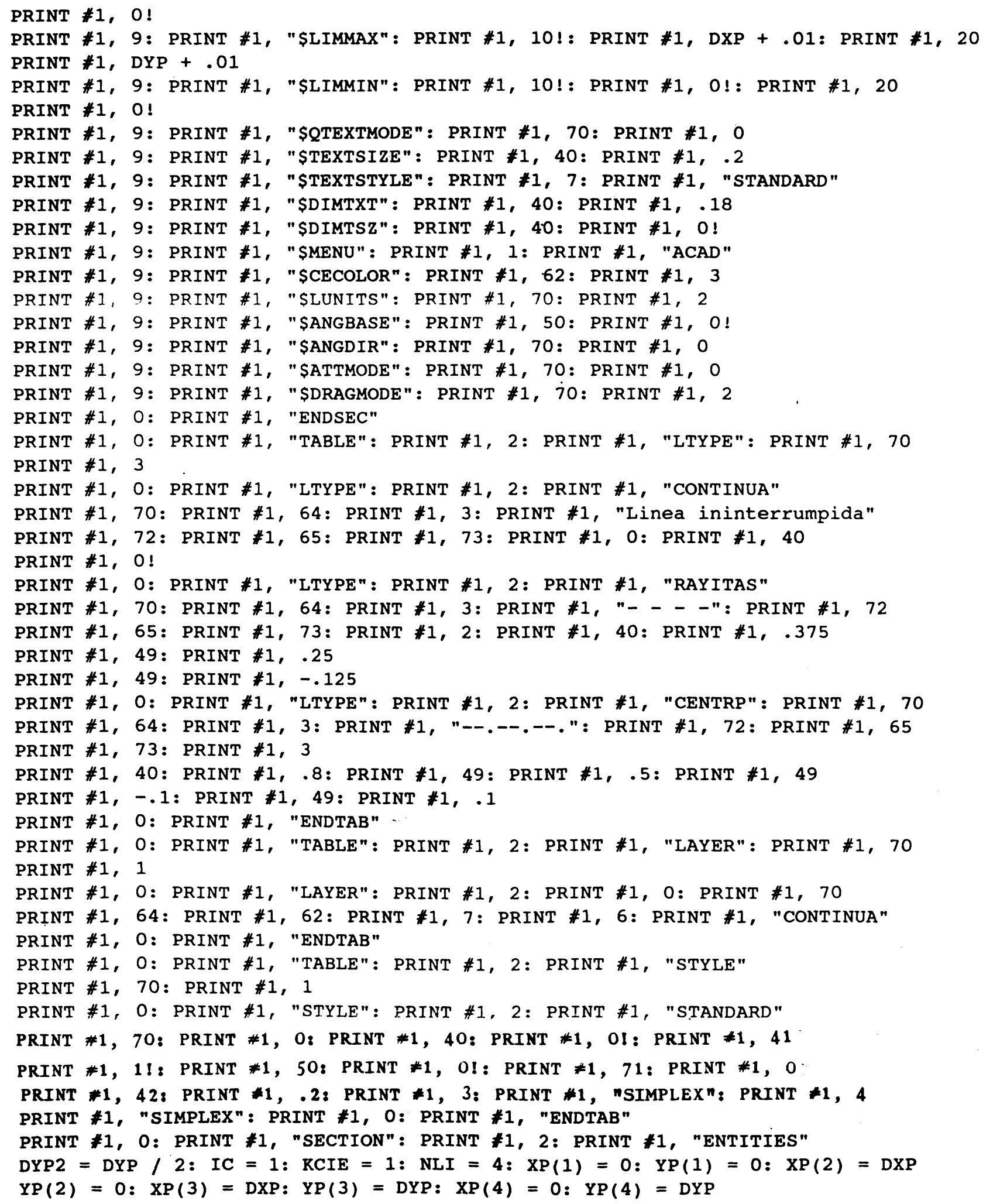


CALL POLI(NLI, $0 !, 0 !, \mathrm{KCIE}, \mathrm{IC}, \mathrm{XP}(), \mathrm{YP}())$

$\mathrm{IC}=3: \mathrm{XP}(1)=3: \mathrm{YP}(1)=.5: \mathrm{XP}(2)=\mathrm{DXP}-.5: \mathrm{YP}(2)=.5: \mathrm{XP}(3)=\mathrm{DXP}-.5$

$\mathrm{YP}(3)=\mathrm{DYP}-.5: \mathrm{XP}(4)=3: \mathrm{YP}(4)=\mathrm{DYP}-.5$

CALL POLI(NLI, $0 !, 0 !$, KCIE, IC, XP(), YP()): IT\$ = "CONTINUA"

CALL LINEA(IC, $0 !, 0 !$, ITS, $0 !$, DYP2, $3 !$, DYP2)

END SUB

SUB LINEA (IC, XDI, YDI, IT\$, X1, Y1, X2, Y2) STATIC

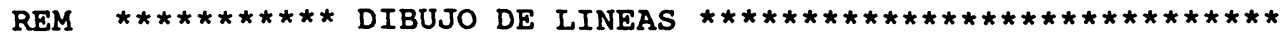

PRINT \#1, 0: PRINT \#1, "LINE": PRINT \#1, 8: PRINT \#1, "0": PRINT \#1, 6

PRINT \#1, ITS: PRINT \#1, 62: PRINT \#1, IC

PRINT \#1, 10: PRINT \#1, X1 + XDI: PRINT \#1, 20: PRINT \#1, Y1 + YDI: PRINT \#1, 11 PRINT \#1, X2 + XDI: PRINT \#1, 21: PRINT \#1, Y2 + YDI

END SUB

SUB POLI (NLI, XDI, YDI, KCIE, IC, XP(), YP()) STATIC

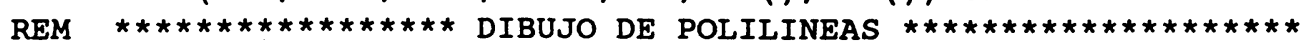

PRINT \#1, 0: PRINT \#1, "POLYLINE": PRINT \#1, 8: PRINT \#1, "0": PRINT \#1, 62

PRINT \#1, IC: PRINT \#1, 66: PRINT \#1, 1: IF KCIE = 1 THEN PRINT \#1, 70

PRINT $1,1:$ NVER $=$ NLI + 1 - KCIE

FOR KV $=1$ TO NVER

PRINT \#1, 0: PRINT \#1, "VERTEX": PRINT \#1, 8

PRINT \#1, "0": PRINT \#1, 62: PRINT \#1, IC: PRINT \#1, 10

PRINT \#1, XP(KV) + XDI: PRINT \#1, 20: PRINT \#1, YP(KV) + YDI

NEXT KV

PRINT \#1, 0: PRINT \#1, "SEQEND": PRINT \#1, 8: PRINT \#1, "0": PRINT \#1, 62

PRINT \#1, IC

END SUB

SUB TEX (IC, XDI, YDI, X1, Y1, HT, T\$) STATIC

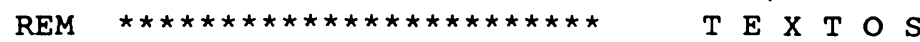

PRINT \#1, 0: PRINT \#1, "TEXT": PRINT \#1, 8: PRINT \#1, "0": PRINT \#1, 62

PRINT \#1, IC: PRINT \#1, 10: PRINT \#1, X1 + XDI: PRINT \#1, 20: PRINT \#1, Y1 + YDI PRINT \#1, 40: PRINT \#1, HT: PRINT \#1, 1: PRINT \#1, TS

END SUB

SUB TEXIN (IC, XDI, YDI, X1, Y1, HT, TS, ANG) STATIC

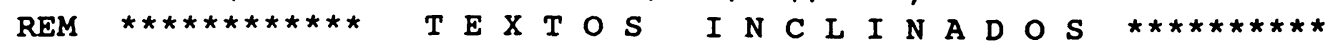

PRINT \#1, 0: PRINT \#1, "TEXT": PRINT \#1, 8: PRINT \#1, "0": PRINT \#1, 62

PRINT \#1, IC: PRINT \#1, 10: PRINT \#1, X1 + XDI: PRINT \#1, 20: PRINT \#1, Y1 + YDI

PRINT \#1, 40: PRINT \#1, HT: PRINT \#1, 1: PRINT \#1, T\$: PRINT \#1, 50

PRINT \#1, ANG

END SUB

DEFSNG I-N

SUB CIRCUL (IC, XDI, YDI, X1, Y1, R) STATIC

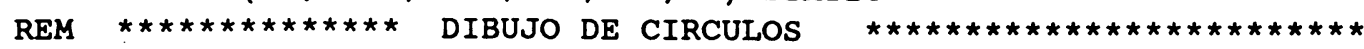

PRINT \#1, 0: PRINT \#1, "CIRCLE": PRINT \#1, 8: PRINT \#1, "0": PRINT \#1, 62

PRINT \#1, IC: PRINT \#1, 10: PRINT \#1, X1 + XDI: PRINT \#1, 20: PRINT \#1, Y1 + YDI PRINT \#1, 40: PRINT \#1, R

END SUB 


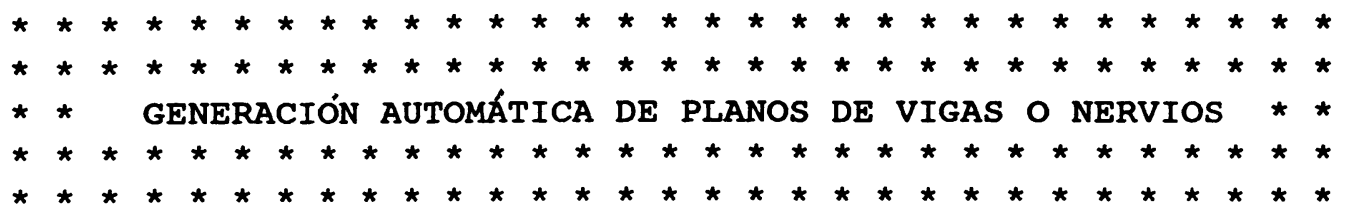

DIM NNC\$ (50)

PRINT : PRINT

INPUT " Se desea obtener planos de vigas (1), o de nervios (2) ";KLAVE OPEN "O",\#1,"C: \ACAD10\DXF.SCR"

CALL NUVIG (NNC, NNC\$()) : IF KLAVE=1 THEN NO1\$="C:|VIGAS|" : NO2\$="PV"

IF KLAVE $=2$ THEN NO1\$="C: \NERVIOS $\backslash ":$ NO2\$="PL"

FOR I=1 TO NNC

PRINT \#1,USING "\#"; : NVS=NO2\$+NNC\$(I)+"=" : PRINT \#1,NV\$

PRINT \#1,"DXFIN" : NO\$=NO1\$+NO2\$+NNC\$(I) : PRINT \#1,NO\$ : PRINT \#1,"END" NEXT I

PRINT \#1,"0"

CLOSE 1

END

SUB NUVIG(NNC, NNC\$(1)) STATIC

CLS : LOCATE $4,2,1$

INPUT " Es un bloque de planos consecutivas (S/N): ";KNC\$

IF KNC\$="N" GO TO NN2

IF KNCS="n" GO TO NN2

LOCATE 6,2,1: INPUT " Numero del primer plano: ";NN1

LOCATE $8,2,1$ : INPUT " Numero del ultimo plano: ";NNU

LOCATE $10,2,1$

PRINT " $\mathrm{N}$ u m e ro d e 1 o $s$ p 1 a n o $s$ :"

$\mathrm{NNC}=\mathrm{NNU}-\mathrm{NN} 1+1: \mathrm{LIN}=10: \mathrm{COL}=54$

FOR $I=1$ TO $N N C+1$

$\mathrm{NNO}=\mathrm{NN} 1+\mathrm{I}-1$ : IF $\mathrm{I}=\mathrm{NNC}+1$ THEN $\mathrm{NNO}=0$

NNS=STRS (NNO)

L1 $=\operatorname{LEN}(\mathrm{NN} \$)-1$

$\operatorname{NNCS}(I)=\operatorname{RIGHT} \$(N N S, L 1)$

LOCATE LIN,COL, 1 : PRINT USING "\#\#";NNO

$\mathrm{COL}=\mathrm{COL}+5:$ IF $\mathrm{COL}>76$ THEN LIN=LIN+2 : COL=2

NEXT I

GOTO NN4

NN2 : LOCATE $10,2,1$

PRINT " Numero de los planos (Para terminar, dar 0):"

$L I N=10: C O L=54: N N C=0$

FOR $I=1$ TO 50

LOCATE LIN,COL, 1 : INPUT " "NNC\$(I) : IF NNCS(I)="O" GOTO NN4

$\mathrm{NNC}=\mathrm{NNC}+1: \mathrm{COL}=\mathrm{COL}+5:$ IF $\mathrm{COL}>76$ THEN $\mathrm{LIN}=\mathrm{LIN}+2: \mathrm{COL}=2$

NEXT I

NN4 : END SUB 
116

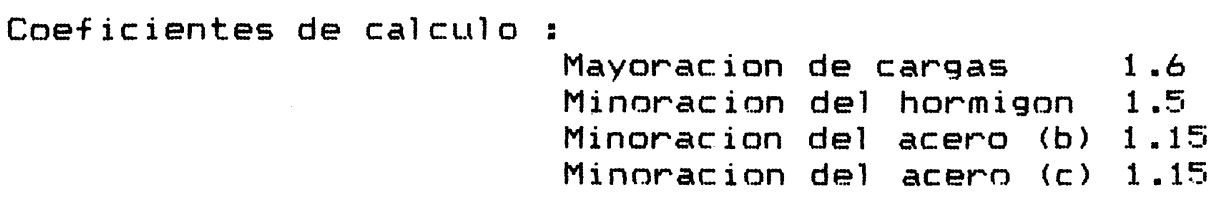

Resistencia de los materiales:

Resistencia caracteristica hormigon 175

Limite el astico acero (b) . . . . . 5100

Tension de rotura acero (b) . . . . 6000

Limite el astico acero (c) . . . . 4830

Tension de rotura acero (c) . . . 6000

\begin{abstract}
II E F I N I C I ON DE LAFGAS
\end{abstract}

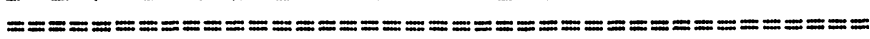

\begin{tabular}{|c|c|c|c|}
\hline CLAVE: & LESIGNACION & CONCAFIGA & SUBRECARGA \\
\hline 1 & VIV & 1000 & 0 \\
\hline 2 & SOTANO & 405 & 400 \\
\hline 3 & BA.JA & 405 & 500 \\
\hline 4 & $E S C$ & 500 & 400 \\
\hline
\end{tabular}
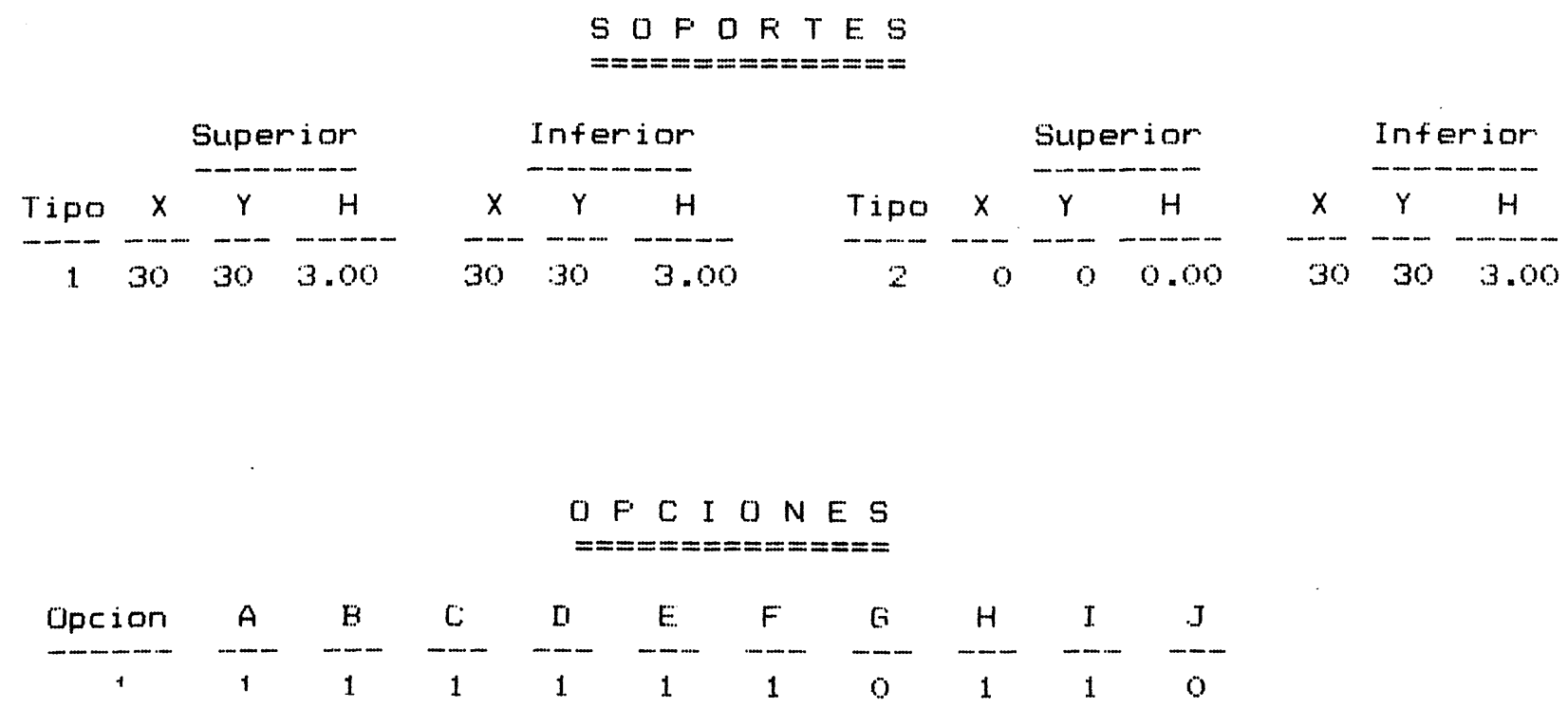


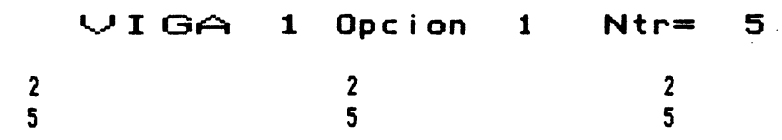

$\left.\sqrt{\frac{40 * 29 / 1}{45 \mathrm{~A}}}\right|_{39} \sqrt{40 * 29 / 1}$

Unif. 1/ 1.68

Punt. 1/ $2.30 / 2.95$

Loc1. $1 / 1.08 / 0.00 / 2.95$

\section{Unif. 1/ 1.68}

Punt. 1/ 2.30/0.95

Punt. 1/ $2.30 / 3.90$

Loc1. $1 / 1.08 / 0.95 / 3.90$

$\prod_{43} \sqrt{40 * 29 / 1}$

Unif. $1 / 1.68$

Punt. 1/ 2.30/ 1.45

Punt. $1 / 2.30 / 4.35$

Loc1. 1/ 1.08/ 1.45/ 4.35

$\left.\sqrt{47}_{50 * 29 / 1}^{4.65}\right|_{51} \sqrt{5.00 * 29 / 1}$

Unif, $1 / 1.68$

Punt. 1/ 2.30/ 1.90

Unif. 1/ 1.68

Loc1. $1 / 1.08 / 2.30 / 5.00$

UIGA 2 Opcion 1 Ntr= 3

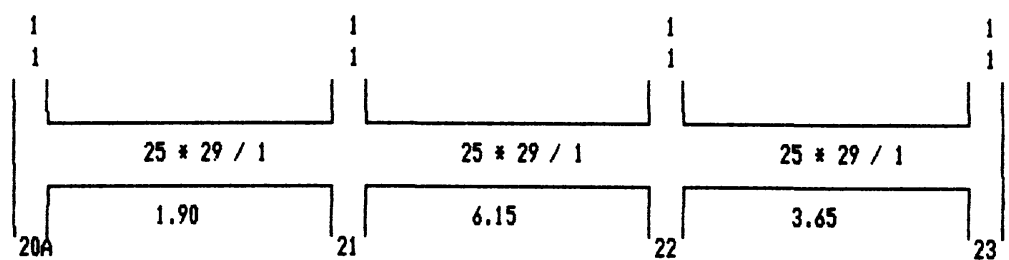

Unif, $1 / 1.05$

Unif. 1/ 1.05

Unif, 1/ 1.05

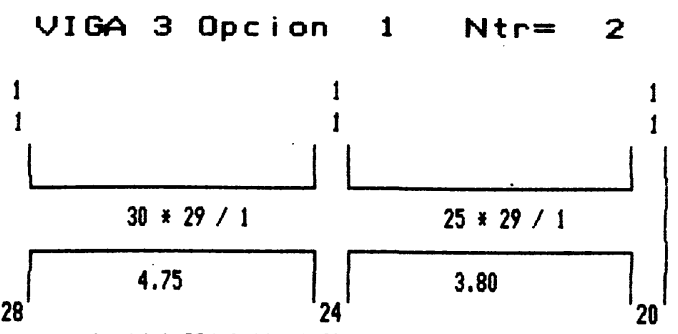

Locl. 1/ 1.93/ 0.00/4.75 Unif, 1/ 0.50

Punt. 1/ 1.08/ 3.65 


\section{GA 1}

CORTANTES

MOMENTOS FLECTORES

UANO LUZ FLECHA I200A DRCHA $0 \quad 0.1 * \mathrm{~L} \quad 0.2 * \mathrm{~L} \quad 0.3 * \mathrm{~L} \quad 0.4 * \mathrm{~L} \quad 0.5 * \mathrm{~L} \quad 0.6 * \mathrm{~L} \quad 0.7 * \mathrm{~L} \quad 0.8 * \mathrm{~L} \quad 0.9 * \mathrm{~L} \quad \mathrm{~L}$

$\begin{array}{lllllllllllllllll}1 & 4.20 & 0.3 & \text { SUP. } & 6.0 & -7.8 & -1.7 & 0.6 & 2.3 & 3.4 & 4.0 & 4.1 & 3.7 & 2.7 & 0.3 & -2.5 & -5.6\end{array}$

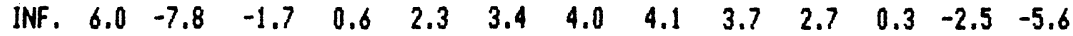

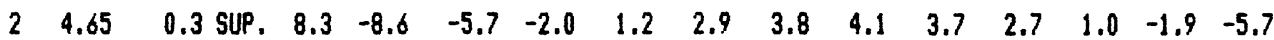

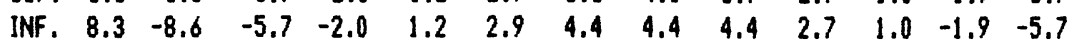

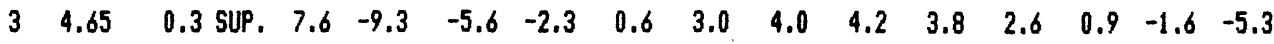

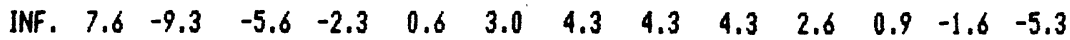

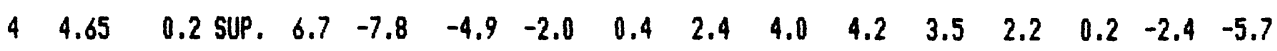

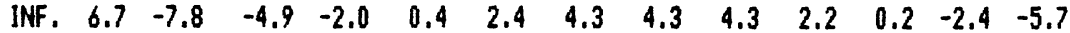

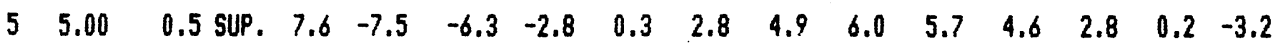

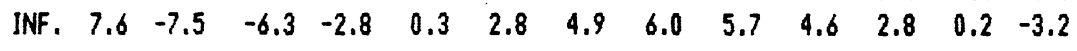

W GA こ

\section{CORTANTES}

MOMENTOS FLECTORES

UANO LUZ FLECHA IZODA DRCHA

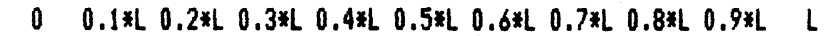

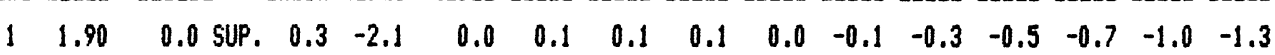

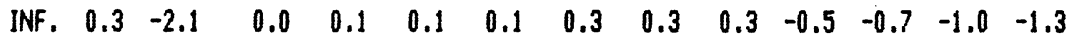

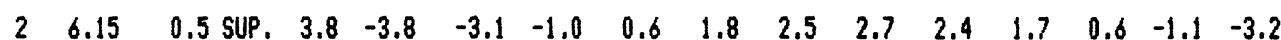
$\begin{array}{llllllllllllll}\text { INF. } 3.8 & -3.8 & -3.1 & -1.0 & 0.6 & 1.8 & 2.6 & 2.7 & 2.6 & 1.7 & 0.6 & -1.1 & -3.2\end{array}$

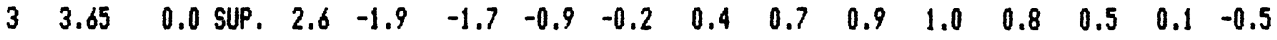

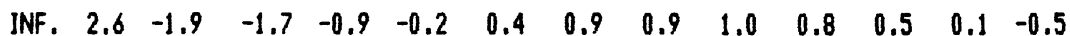

1I GA B

CORTANTES MOMENTOS FLECTORES

UANO LUZ FLECHA IZODA DRCHA 0 0.1*L $0.2 * \mathrm{~L} \quad 0.3 * \mathrm{~L} \quad 0.4 * \mathrm{~L} \quad 0.5 * \mathrm{~L} \quad 0.6 * \mathrm{~L} \quad 0.7 * \mathrm{~L} \quad 0.8 * \mathrm{~L} \quad 0.9 * \mathrm{~L} \quad \mathrm{~L}$

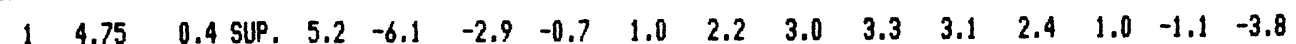
INF. $\begin{array}{lllllllllllll}5.2 & -6.1 & -2.9 & -0.7 & 1.0 & 2.2 & 3.0 & 3.3 & 3.1 & 2.4 & 1.0 & -1.1 & -3.8\end{array}$

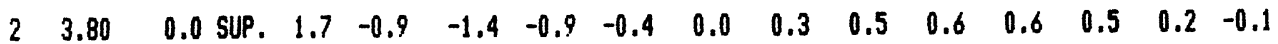

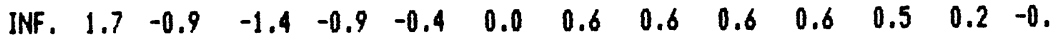

$11 \leqslant>0$

A R M A U R A S

$\begin{array}{lllllllllllll}0 & 0.1 * L & 0.2 * L & 0.3 * L & 0.4 * L & 0.5 * L & 0.6 * L & 0.7 * L & 0.8 * L & 0.9 * L & L\end{array}$

$\begin{array}{lllllllllll}3.25 & 0.00 & 0.00 & 0.00 & 0.00 & 0.00 & 0.00 & 0.00 & 0.00 & 3.70 & 9.36\end{array}$ $\begin{array}{lllllllllll}0.00 & 3.25 & 3.36 & 5.26 & 6.34 & 6.48 & 5.66 & 3.98 & 3.25 & 0.00 & 0.00\end{array}$

$\begin{array}{lllllllllll}9.53 & 3.25 & 0.00 & 0.00 & 0.00 & 0.00 & 0.00 & 0.00 & 0.00 & 3.25 & 9.62\end{array}$ $\begin{array}{llllllllllll}0.00 & 0.00 & 3.25 & 4.30 & 7.02 & 7.02 & 7.02 & 4.09 & 3.25 & 0.00 & 0.00\end{array}$

$\begin{array}{lllllllllll}9.42 & 3.43 & 0.00 & 0.00 & 0.00 & 0.00 & 0.00 & 0.00 & 0.00 & 3.25 & 8.73\end{array}$ $\begin{array}{lllllllllll}0.00 & 0.00 & 3.25 & 4.56 & 6.91 & 6.91 & 6.91 & 3.96 & 3.25 & 0.00 & 0.00\end{array}$

$\begin{array}{lllllllllll}8.02 & 3.25 & 0.00 & 0.00 & 0.00 & 0.00 & 0.00 & 0.00 & 0.00 & 3.54 & 9.52\end{array}$ $\begin{array}{llllllllllll}0.00 & 0.00 & 3.25 & 3.63 & 6.73 & 6.73 & 6.73 & 3.25 & 3.25 & 0.00 & 0.00\end{array}$

$\begin{array}{lllllllllll}11.02 & 4.20 & 0.00 & 0.00 & 0.00 & 0.00 & 0.00 & 0.00 & 0.00 & 0.00 & 4.82\end{array}$ $\begin{array}{lllllllllll}0.00 & 0.00 & 3.25 & 4.25 & 7.95 & 10.23 & 9.56 & 7.41 & 4.19 & 3.25 & 0.00\end{array}$

$11 \leq 6$

A R M A D U R A S

$\begin{array}{llllllllll}0 & 0.1 * \mathrm{~L} & 0.2 * \mathrm{~L} & 0.3 * \mathrm{~L} & 0.4 * \mathrm{~L} & 0.5 * \mathrm{~L} & 0.6 * \mathrm{~L} & 0.7 * \mathrm{~L} & 0.8 * \mathrm{~L} \quad & 0.9 * \mathrm{~L} \quad L\end{array}$ $\begin{array}{lllllllllll}0.00 & 0.00 & 0.00 & 0.00 & 0.00 & 2.03 & 2.03 & 2.03 & 2.03 & 2.03 & 2.03\end{array}$ $\begin{array}{lllllllllll}0.00 & 2.03 & 2.03 & 2.03 & 2.03 & 2.03 & 2.03 & 0.00 & 0.00 & 0.00 & 0.00\end{array}$

$\begin{array}{lllllllllll}5.03 & 2.03 & 0.00 & 0.00 & 0.00 & 0.00 & 0.00 & 0.00 & 0.00 & 2.03 & 5.23\end{array}$ $\begin{array}{llllllllllll}0.00 & 0.00 & 2.03 & 2.66 & 4.13 & 4.24 & 4.13 & 2.59 & 2.03 & 0.00 & 0.00\end{array}$

$\begin{array}{lllllllllll}2.59 & 2.03 & 2.03 & 0.00 & 0.00 & 0.00 & 0.00 & 0.00 & 0.00 & 0.00 & 2.03\end{array}$ $\begin{array}{lllllllllll}0.00 & 0.00 & 0.00 & 2.03 & 2.03 & 2.03 & 2.03 & 2.03 & 2.03 & 2.03 & 0.00\end{array}$ 11 s 10

A R MA D URAS

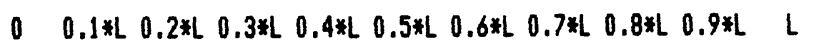
$\begin{array}{lllllllllll}4.60 & 2.44 & 0.00 & 0.00 & 0.00 & 0.00 & 0.00 & 0.00 & 0.00 & 2.44 & 6.20\end{array}$ $\begin{array}{lllllllllll}0.00 & 0.00 & 2.44 & 3.40 & 4.71 & 5.22 & 4.83 & 3.62 & 2.44 & 0.00 & 0.00\end{array}$

$\begin{array}{lllllllllll}2.13 & 2.03 & 2.03 & 0.00 & 0.00 & 0.00 & 0.00 & 0.00 & 0.00 & 0.00 & 2.03\end{array}$ $\begin{array}{lllllllllll}0.00 & 0.00 & 0.00 & 2.03 & 2.03 & 2.03 & 2.03 & 2.03 & 2.03 & 2.03 & 0.00\end{array}$ 

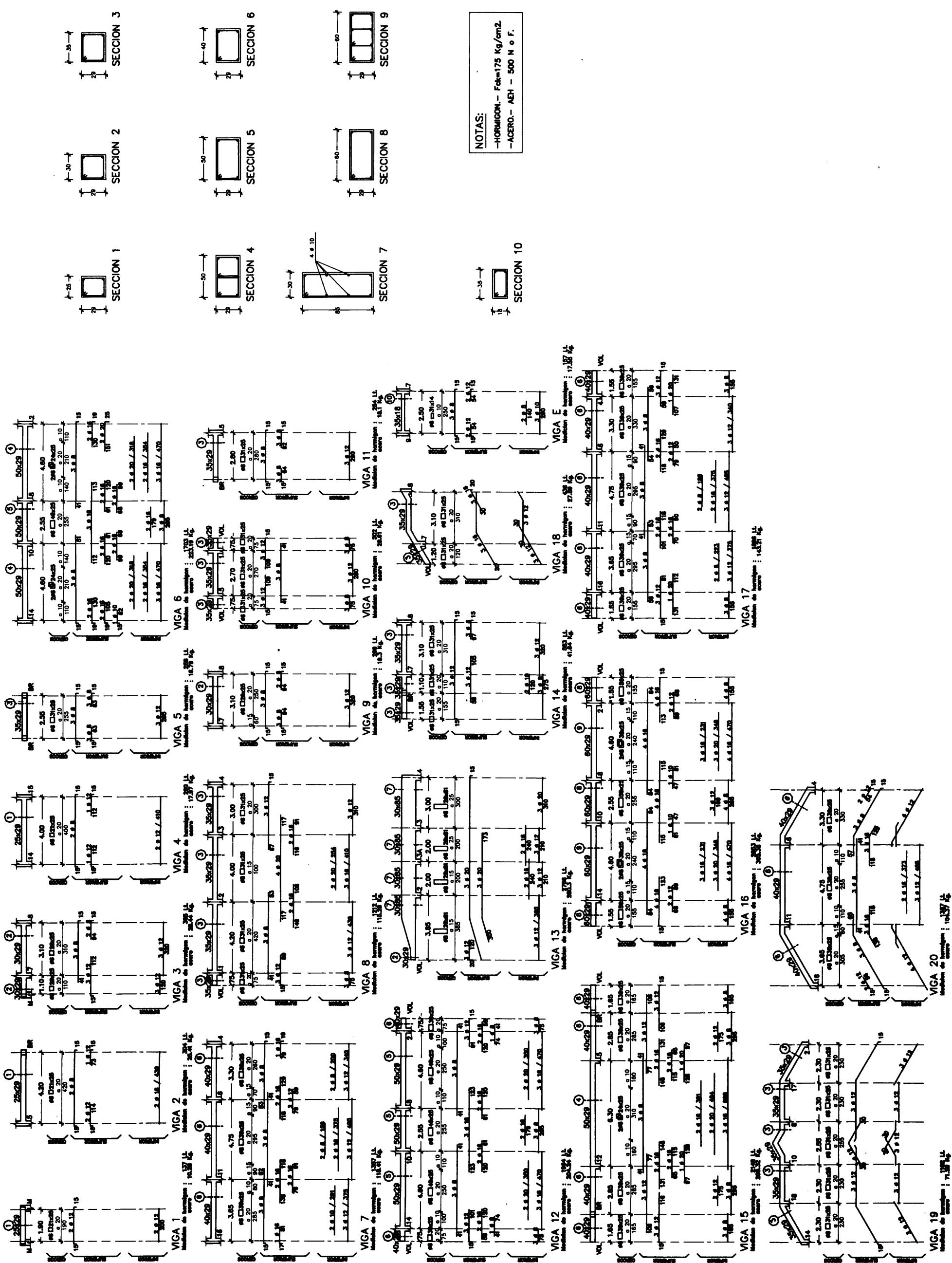


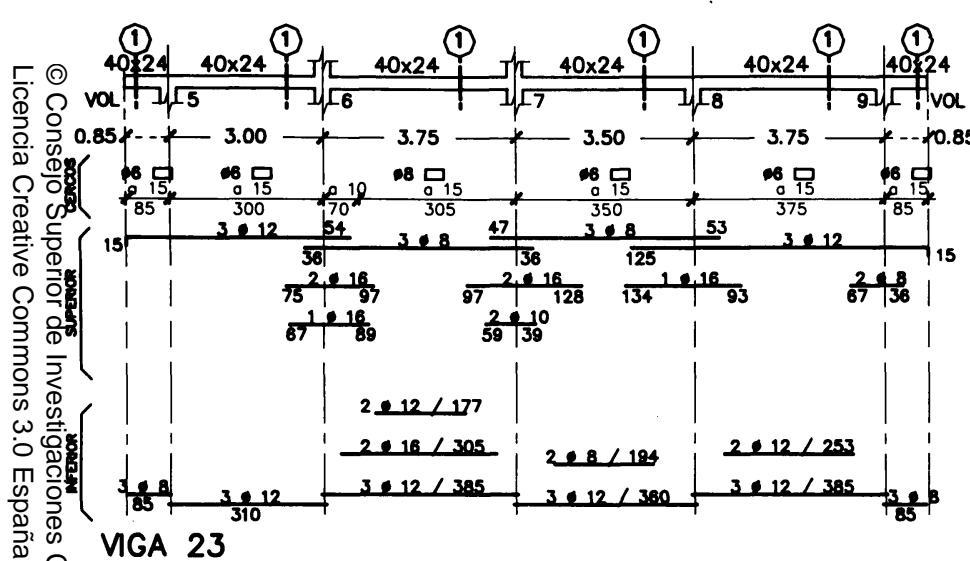

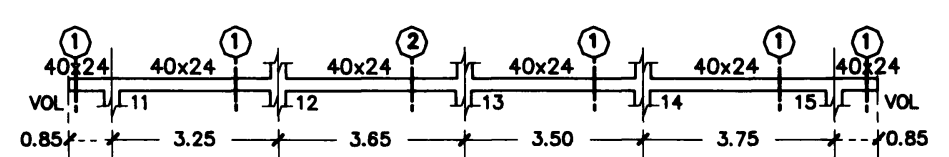

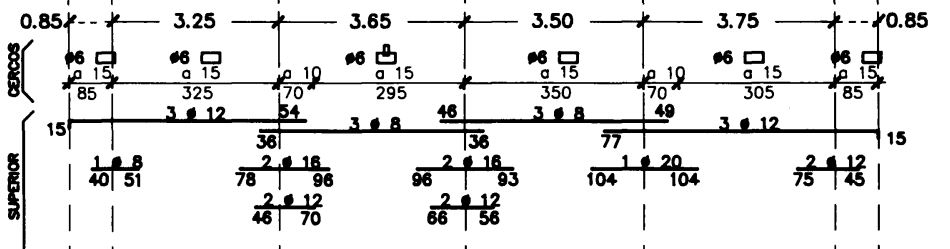

$\underset{\text { SECCION } 1}{\stackrel{4}{24}+40-\infty}$

$\underset{\text { SECCION } 2}{+40 \rightarrow+}$

\section{VIGA 23}

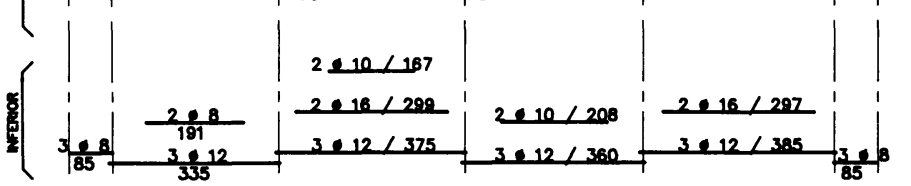

VIGA 24

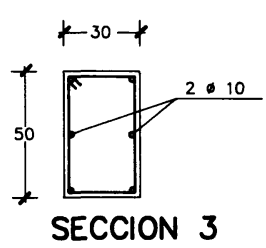

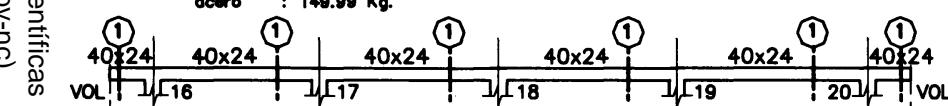
$0.85-3.25 \longrightarrow 3.50 \longrightarrow 3.50 \longrightarrow 3.75 \longrightarrow-10.85$

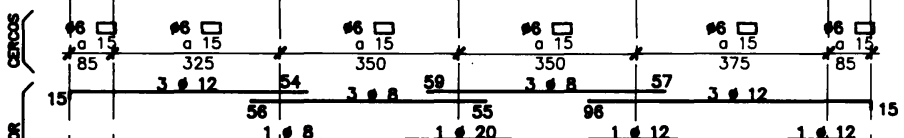

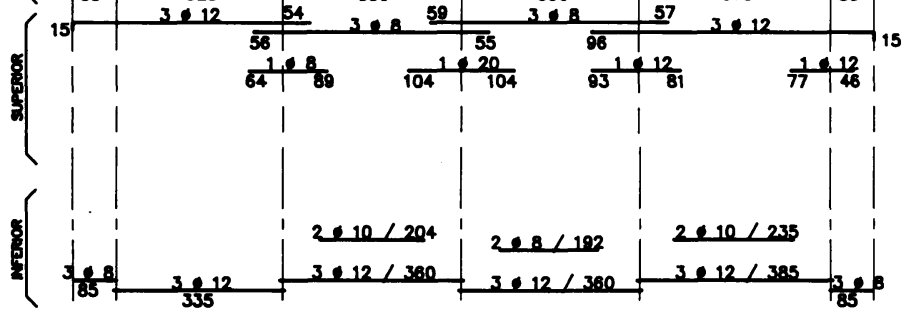

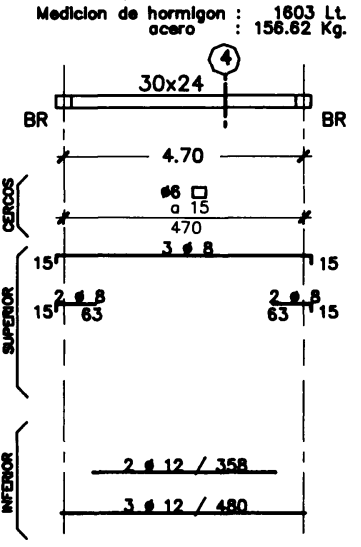

VGA 27

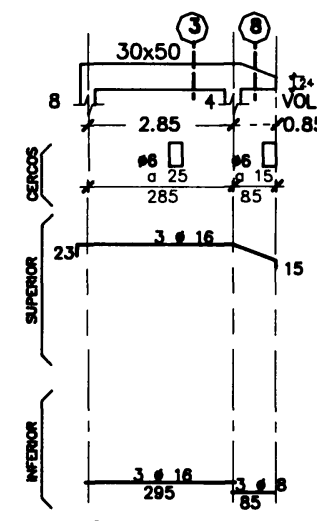

VGA 28
VIGA 25

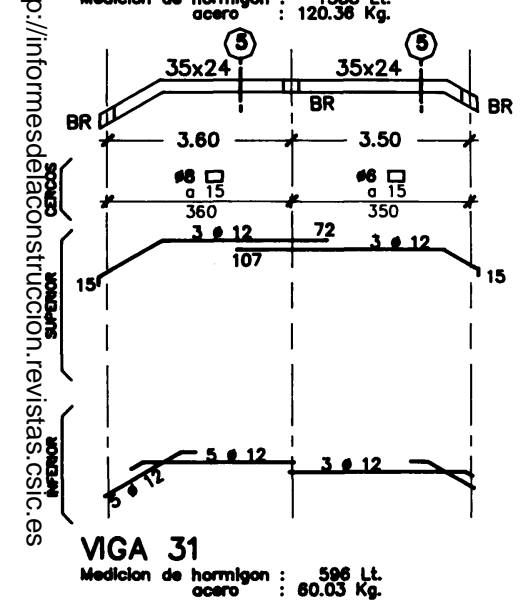

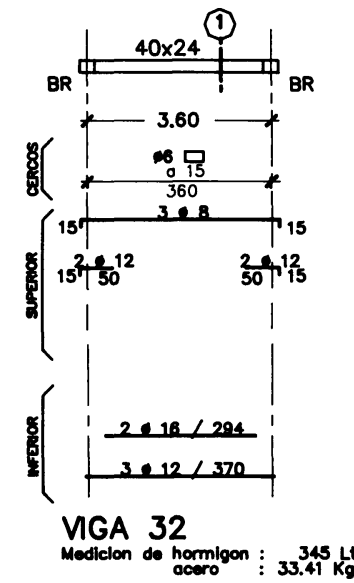

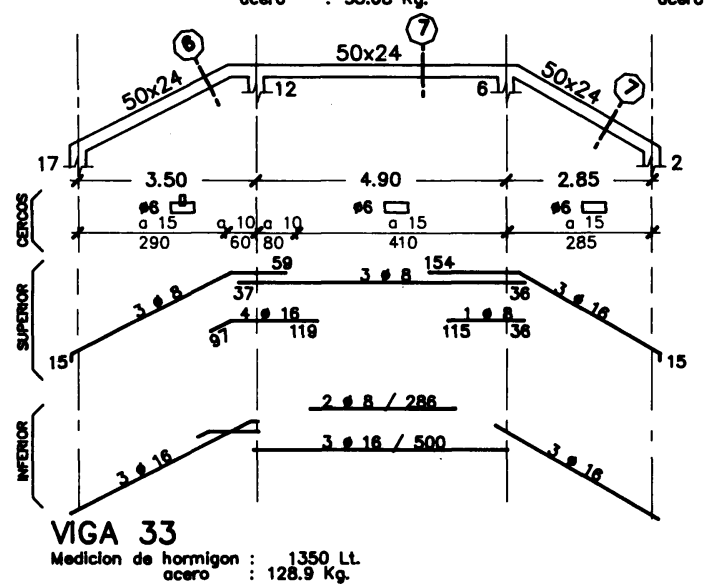

$\frac{\prod_{\text {SECCION } 5}^{+35-k}}{\square}$

$\underset{24}{24} \square$

SECCION 6

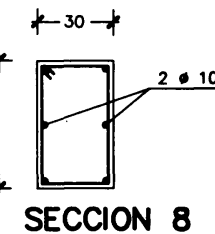

ए

SECCION 4

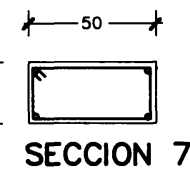

\section{NOTAS:}

-HORMIGON. - Fck=175 kg/cm2.

-ACERO.- AEH-400 N O F

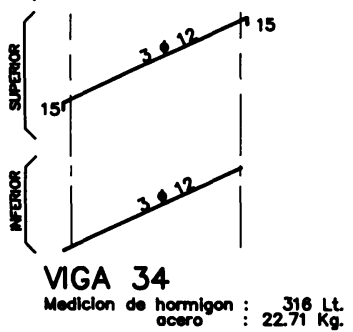

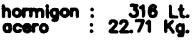

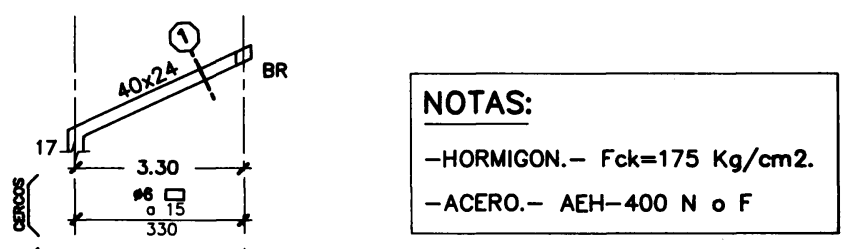

